\title{
Preparation and Evaluation of Inflammation Targeted Nano- micellar Formulation of Celecoxib
}

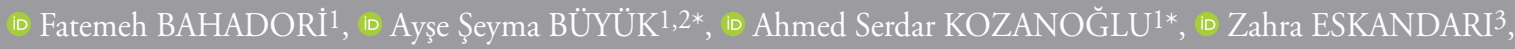

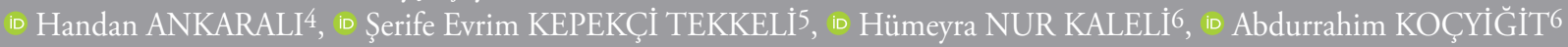

1Bezmialem Vakıf University Faculty of Pharmacy, Department of Pharmaceutical Biotechnology, İstanbul, Turkey

2İstinye University Faculty of Pharmacy, Department of Clinical Pharmacy, İstanbul, Turkey

${ }_{3}^{3} Y 11$ diz Technical University Faculty of Sciences and Arts, Department of Chemistry, Biochemistry Division, İstanbul, Turkey

4 İstanbul Medeniyet University Faculty of Medicine, Department of Biostatistics and Medical Informatics, İstanbul, Turkey

${ }_{5}^{5}$ Bezmialem Vakıf University Faculty of Pharmacy, Department of Analytical Chemistry, İstanbul, Turkey

${ }_{6}^{6}$ Bezmialem Vakıf University Faculty of Medicine, Department of Medicinal Biochemistry, İstanbul, Turkey

\section{ABSTRACT}

Objective: Celecoxib (CLX), brand named Celebrex, which belongs to non-steroidal anti-inflammatory drugs family, selectively inhibits the cytokine related cyclooxygenase-2 isoenzyme and thus, possesses less gastrointestinal side effects, have shown to cause stroke, myocard infarction and even death in some cases. In this study we aimed to target inflammation site using CLX uploaded nano-micelles (nano-CLX) made of poly (lactic-co-glycolic) acid (PLGA) to protect other tissues from its side effects.

Methods: CLX was physically entrapped in PLGA micelles using w/o/w emulsion method, resulted in obtaining mono-dispersed particles with $112 \mathrm{~nm}$ size. $50 \mathrm{mg}$ PLGA was able to carry $50 \mathrm{mg}$ CLX in $20 \mathrm{~mL}(2.5 \mathrm{mg} / \mathrm{mL})$ with encapsulation efficiency of $85 \%$. Rheumatoid arthritis model was achieved by injection of complete Freund's Adjuvant to the hint paw of Wistar rats. Infected groups received oral Celebrex, intravenous (i.v.) Celebrex and nano-CLX. Each group was compared with a healthy control group receiving the drug via the same routes. The obtained serums and the hint paw sizes were studied for 6 hours in 3 time periods.

Results: Prostaglandin E2 and tumor necrosis factor- $\alpha$ levels were found to be decreased for longer time period by application of nano-CLX compared to oral and i.v. Celebrex. Interleukin-1 (IL-1) and IL-6 levels showed a dramatic decrease at orally administered Celebrex groups, showing the accumulation of these pro-inflammatory factors at inflammation area.

Conclusion: Based on the hypothesis that the ratio of blood parameters is inversely proportional to accumulation at inflammation site, thus, our nano-formulation is targeted to the tissues in the systemic blood flow and have a better selective inhibition.

Keywords: Targeted inflammation therapy, Celecoxib, nano drug delivery system, PLGA, COX2

\section{Introduction}

Rheumatoid arthritis (RA) is a multisystem chronic inflammatory disease which especially affects the peripheral joints in a symmetric manner and often causes bone erosions, cartilage destruction and joint deformities. RA has a worldwide distribution, with a prevalence of approximately $1 \%$. Major complaint is pain and it is aggravated with movement (1). Preferably, non-steroidal anti-inflammatory drugs (NSAIDs) are used, which tend to control the symptoms by blocking cyclooxygenase-2 (COX-

*: These authors contributed equally to this work

Address for Correspondence: Fatemeh BAHADORI, Bezmialem Vakıf University Faculty of Pharmacy, Department of Pharmaceutical Biotechnology, İstanbul, Turkey

Phone: +90 5342912494 E-mail: fatemehbahadori@gmail.com ORCID ID: orcid.org/0000-0003-4224-9309

Cite this article as: Bahadori F, Büyük AŞ, Kozanoğlu AS, Eskandarı Z, Ankaralı H, Kepekçi Tekkeli ŞE, Kaleli HN, Koçyiğit A. Preparation and Evaluation of Inflammation Targeted Nano-micellar Formulation of Celecoxib. Bezmialem Science 2019;7(3):208-14. 
2) and the production of the thromboxanes, prostacyclins and prostaglandins. Gastrointestinal (GI) intolerance and the cardiovascular complications are the common side-effects of the NSAIDs and COX-2 inhibitors (1).

Celecoxib (Celebrex ${ }^{\circ}$ (CLX), a selective COX-2 inhibitor, was approved by the FDA for the treatment of chronical polyarthritis and osteoarthritis in 1998 (2). Certainly, selective COX2 inhibitors decrease the risk of the GI side effects. Nevertheless, COX-2 is not only a pro-inflammatory inducible enzyme, it also has various physiological functions which means it is constitutively expressed to a high extent in the human body (3). The clarified paradigm of constitutive COX-1 and inducible COX-2 has several exceptions: COX-1 may be modulated during development, whereas COX-2 is constrictively expressed in the kidney, brain and reproductive tissues. Additionally, selective inhibitors of COX-2 decrease prostacyclin, an atheroprotective agent, however it does not decrease COX-1 derived thromboxane $A_{2}$, a vasoconstrictor and proaggregatory mediator, which can predispose patients to stroke and heart attack. Therefore, the use of CLX requires further improvement (4).

Nano-technology is a science area dedicated to the construction, design and utilization of functional structures on the nanometer scale (often $100 \mathrm{~nm}$ or even smaller). Biodegradable nanoparticles are regularly used to improve the therapeutic value of different water soluble/insoluble medicinal drugs and bioactive molecules by improving their retention time, solubility, bioavailability or by bringing the targeting property to the small molecules such as CLX. These nanoparticle-drug formulation lowers the expenses and risks of toxicity in patients (5). Enhanced Permeability and Retention (EPR) are the most known phenomena in targeting disease site using nano-drug delivery systems (NDDS). This elegant strategy uses the enhanced gap between the endothelial cells of veins at site of inflammation or cancer compared to that of healthy tissues (6). This enhanced gap reaches to around 200 $\mathrm{nm}$, while it has been measured as $10-15 \mathrm{~nm}$ in healthy tissue. Entrapping the small molecules within NDDS with size less than $200 \mathrm{~nm}$ provides accumulation of drug molecules only at the inflammation site.

In order to transport the drug molecules to the cells and enzymes, a lot of biodegradable polymers are synthesized $(7,8)$. Polyesters and co-polyesters of the alpha, beta and gamma-hydroxy acids are the most common synthetic biodegradable polymers in the last 20 years. Poly-lactic acid, poly-glicolic acid and Poly (lactic-coglycolic) acid (PLGA), which are copolymer molecules, are the best defined polymers based on to their activities and the designs $(9,10)$. PLGA is accepted as gold standard and is known for selfassembling in aqueous media entrapping the hydrophobic drug molecules at the core part of the formed spherical micelles. PLGA is converted into the lactic acid and the glycolic acid by hydrolysis in the body, thus is bio-compatible and bio-degradable (11).

The aim of this study was prepare and characterize a novel PLGA- CLX nano micellar formulation in order to target the inflammation site to provide high efficacy and low toxicity.

\section{Methods}

\section{Materials}

Complete Freund's Adjuvant (\#F5881), Poly-(D,L-lactideco-glycolide) (\#P2192) and CLX (\#PZ0008) was purchased from Sigma Aldrich, Rat tumor necrosis factor (TNF)- $\alpha$ Kit (\#YHB20170406928), Rat PG-E2 Kit (\#YHB20170406931), Rat interleukin (IL)-1 $\beta$ Kit (\#YHB20170406929) and Rat IL-6 Kit (\#YHB20170406930) was purchased from Yehua, all other chemicals were analytical grade, unless mentioned.

\section{Preparation of the Nano-celecoxib Formulation}

Fifty mg PLGA and increasing amounts of CLX (10-60 mg) were dissolved in $5 \mathrm{~mL}$ of acetone. Acetone solutions were then combined and added wisely by dripping on $20 \mathrm{~mL}$ of d.d. water containing $0.005 \%$ tween 80 in a very slow manner while stirring on magnetic stirrer (12). Stirring was continued overnight allowing all acetone to be evaporated and the day after the mixture's volume was adjusted to $20 \mathrm{~mL}$ and particle size was measured as below. This process is summarized in Figure 1.

\section{Particle Size Measurements}

The sizes of PLGA-CLX and free PLGA particles were measured directly on Zetasizer Nano ZS (model ZEN 3600; Malvern Instrument, Inc., London, UK) at $25^{\circ} \mathrm{C}$. Default setting on the Zetasizer Nano ZS was used, i.e. refractive index, absorption. The dispersant used was water and measurement angle was 173. Measurements were repeated 5 times, 3 minutes each and data were analyzed by number distributions (13). All samples were measured in triplicate. Presence of aggregates in micron size indicated the precipitated drug molecules. The max drug concentration with mono-modelar particle distribution was chosen as the optimized formulation. Further studies were carried out using the optimized formulation. According to our experiences, in case of presence of traces of amorphous aggregates in the system, measurements based on the volume and intensity distributions reveal these aggregates better than the ones based on number distribution. Therefore, all above mentioned measurements were conducted to make sure the obtained toxicity results were caused by the micelles, not aggregates (12).

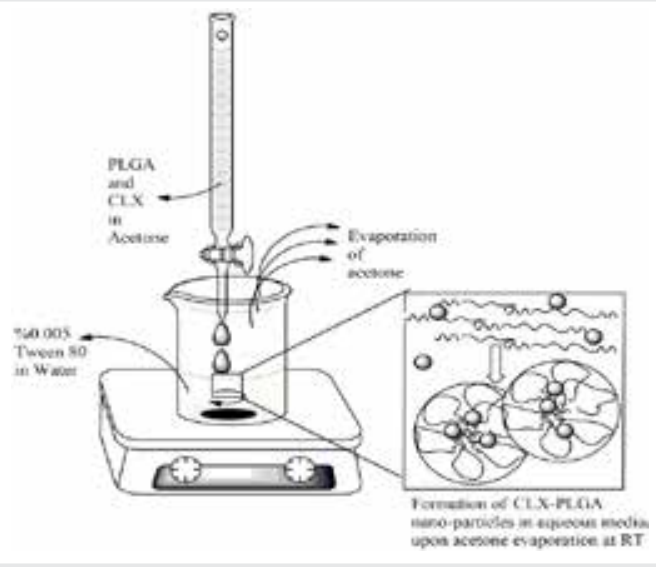

Figure 1. Preparation of CLX-PLGA nano-particles CLX: Celecoxib, PLGA: Poly (lactic-co-glycolic acid) 


\section{In vivo Experiment Process}

The approval for animal experiments was obtained from Bezmialem Vakıf University Experimental Animal Ethics Committee, numbered 2016-241. Since this study was aimed to compare the toxicity of commercially available CLX formulation with the novel nano PLGA-CLX, the toxic dose of CLX was chosen to use in the animal study. It has been demonstrated that 5 and $7 \mathrm{mg} / \mathrm{kg}$ CLX is tolerable, thus $10 \mathrm{mg} / \mathrm{kg}$ of CLX was chosen to be evaluated at all samples.

Thirty two 5-week-old female Wistar rats (Bezmialem Vakıf University Experimental, Animal Research Center, IstanbulTurkey) were separated randomly within 8 weight-matched groups consisted of 4 animals at each group and were given free access to a standard chow and water. All process followed were in complete agreement with The ARRIVE guidelines (14). $0.1 \mathrm{~mL}$ Complete Freund's Adjuvant (F5881) was injected to the right hint paw of the infected groups to induce RA model. At 0 point (before Freud's adjuvant injection), $30 \mathrm{~min}, 3^{\text {rd }}$ and $6^{\text {th }}$ hours after Freud's adjuvant injection, $0.5 \mathrm{~mL}$ blood was taken from all animals and their right hint paw size to which the adjuvant was injected was measured. First group was "Healthy Control Group" (HC) which received neither adjuvant nor medication. Second group, receivedthe adjuvant without medication [RA Control Group rheumatoit artritis control group (RAC)]. The third group received commercially available celebrex formulation orally, dissolved in water without getting infected by the adjuvant (HO) and fourth group received oral celebrex while treated with adjuvant rheumatoit artritis model (RAO). In the same manner, the fifth and sixth groups received CLX intravenously, to compare the efficacy of commercial product while receiving intravenously, and the sixth group was treated with the adjuvant (group 5: healthy rats treated with intravenous and group 6: rheumatoit artritis model treated with intravenous). The last two groups received nano PLGA-CLX formulation intravenously (group 7: home non-invasive ventilation and group 8: RANIV). Oral and intravenöz formulations were applied as max. 0.5 and 1 $\mathrm{mL}$, respectively (the dose was equal to $10 \mathrm{mg} / \mathrm{kg}$ of each animal).

\section{Determination of Cytokines and Prostaglandins in Blood Circulation}

All the blood samples were transferred to empty tubes and were centrifuged in $4000 \mathrm{rpm}$ for 10 minutes to obtain the serum samples. Obtained serum samples were kept at $-80{ }^{\circ} \mathrm{C}$ till the test day. Cytokine and prostaglandin levels in blood were measured by sandwich enzyme-linked immunosorbent assay (ELISA), using IL-1 $\beta$, IL-6, TNF- $\alpha$, prostaglandin E2 (PGE2) ELISA kits. Colorimetric absorbance was measured at $450 \mathrm{~nm}$ on a BioTek ELISA Synergy-H1 microplate reader.

\section{Release Study}

Three mL CLX-PLGA with optimum drug and polymer ratio was transferred into dialysis membrane tubing SPECTRA/POR 3 Dialysis Membrane (Molecular weight cut-off 3,5 Lot No: 9200373) which then placed into $300 \mathrm{~mL}$ of dialysis medium (Phosphate-buffered saline buffer, $\mathrm{pH} 7.4$ ) at $37^{\circ} \mathrm{C}$, while the media was stirred in a slow manner (15). One $\mathrm{mL}$ samples were withdrawn from the dialysis media at $0.5,1,2,3,4,6,8,10,12$, 20, 22 and 24 hours of the experiment and CLX concentration was measured using high performance liquid chromatography (HPLC).

\section{Measuring CLX concentration using HPLC}

An analytical method for measuring CLX at HPLC was developed in order to measure the amount of drug incorporated with PLGA micelle, and to follow the release results. HPLC trials were conducted on a Shimadzu LC 20 liquid chromatograph equipped with a LC-20AT pump, SIL AT-HT auto-sampler part, and ultraviolet detector, which was set at $260 \mathrm{~nm}$ and CLX was passed through the colon with $1 \mathrm{~mL} / \mathrm{min}$ speed with acetonewater (70:30) solution system from the C18 type colon with 4.6 $\mathrm{cm}$ inner diameter, $5 \mu \mathrm{m}$ particle size (Shimadzu; Kyoto, Japan), where the colon heat was stabilized to $25^{\circ} \mathrm{C}$. The CLX retention time was 5 min under these conditions $(16,17)$.

\section{Drug Loading Capacity}

The concentration of CLX used in the preparation of the nanoformulation process was increased step wisely. Prepared CLXPLGA formulation was incubated at room temperature for two hours after which samples with certain volumes were taken from the formulation and diluted with methanol $1 / 5(\mathrm{~V}: \mathrm{V})$ to obtain free drug. Triple samples from supernatant were injected to the HPLC. Encapsulation Efficiency was measured using Equation $(1: 1)(18)$.

$\mathrm{EE} \%=\frac{\text { Total drug incorporated with PLGA }}{\text { Initial quantity of drug }} \times 100$

\section{Stability Studies}

Obtained optimized PLGA-CLX formulation was frozen at -80 ${ }^{\circ} \mathrm{C}$ overnight following by freeze drying at $-80{ }^{\circ} \mathrm{C}$ and $0.03 \mathrm{mbar}$ (lyophilizer, Labconco, Freezone 2.5 plus). The obtained fluffy material was incubated at either room temperature or at $-20^{\circ} \mathrm{C}$ for 1 week, 2 and 6 months, after which all were re-hydrated with the amount of water equal to that of pre-drying. Re-hydrated formulations were studied for particle size and CLX content as a factor of stability, using above mentioned methods (15).

\section{Statistical Analysis}

Regarding hint paw size and cytokine and prostaglandin measurements, each group at same time periods was compared separately and analyzed using Kruskal Wallis variance analysis. Different groups were determined using post hoc Dunn test. The $\mathrm{p}$ value $<0.05$ was considered as statistically significant. All statistical analyzes were done by using SPSS package program (ver.23) (19).

\section{Results}

\section{Particle Size of the CLX Loaded PLGA Micelles}

Particle sizing results of CLX-PLGA are listed in Table 1. As it is seen in Number, volume and intensity results, particle size 
is monomodelar up to $30 \mathrm{mg} / \mathrm{mL}$ CLX. Excess amounts of CLX uploading causes obtaining bimodelar particles. In case of application of nano-CLX-PLGA for studying efficacy of this drug, this ratio could be used as the optimized formulation. However, in this study, we tried to use the toxic dose which corresponded to $10 \mathrm{mg} / \mathrm{kg}$ animal (each rat is around $250 \mathrm{~g}$ ). Thus, we used $50 \mathrm{mg}$ CLX in $50 \mathrm{mg}$ PLGA/20 mL which the ratio of aggregate was less than $10 \%$ in terms of volume and intensity distribution and the particle size distribution was exactly $100 \%$ in terms of number distribution. Figure 2 shows the particle characteristics of the optimized formulation in which the phenylene diisocyanide is 0.415 and the majority of particles are less than $200 \mathrm{~nm}$. It is noteworthy that all particles were smaller than $200 \mathrm{~nm}$, which was necessary for a particle to be used in EPR effect (6).

\section{Hint Paw Size Measurement Results}

Figure 3 shows the increase in the hint paw size of the healthy and infected animals with adjuvant. The differences between the paw size of the healthy groups (encoded with $\mathrm{H}$ as $\mathrm{HC}, \mathrm{HO}$ etc.) and the RA (encoded by RA) groups increased significantly by time. However, this increase in difference was less significant in the RA group treated with nano-CLX-PLGA $(\mathrm{p}<0.01)$.

\section{Evaluation of Cytokines and Prostaglandins In-vivo}

Figure 4 shows the variations in ratios of some biological indicators of inflammation before and after administration of oral and intravenous Celebrex and intravenous application of nano-CLX-PLGA to both healthy animals and RA models.

As it could be seen above, average results of TNF- $\alpha$ at the $6^{\text {th }}$ hour showed meaningful decrease in RANIV group compared to that of RAO $(p<0.05$ or $p<0.10)$, however, orally administered Celebrex was significantly more successful in decreasing the level of PGE2 within 3 hours (not till 6 hours). Nevertheless, intravenously administered celebrex was able to decrease the level of this prostaglandin in the long-term (6 hours). Similarly, RANIV group showed significantly decreased levels of PGE2 in the long-term (6 hours). No significant difference observed

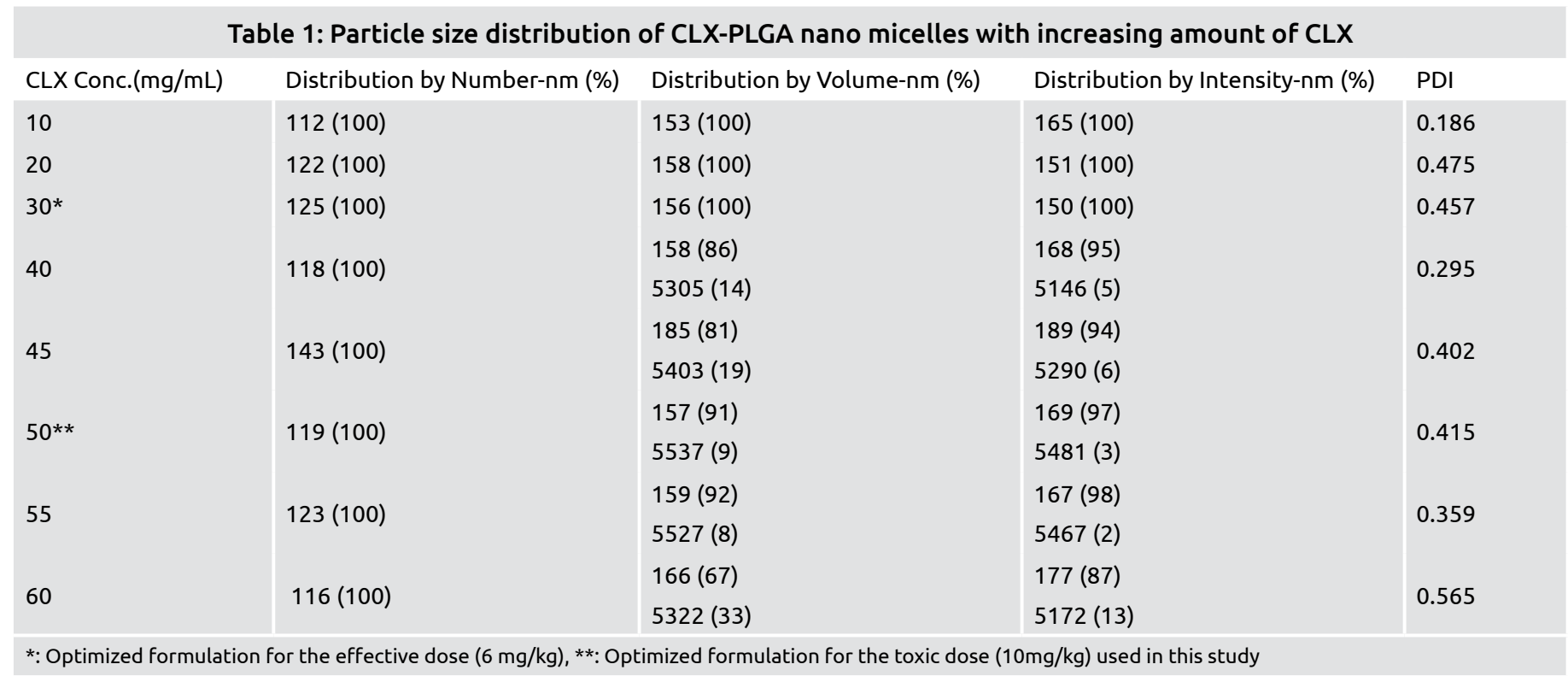

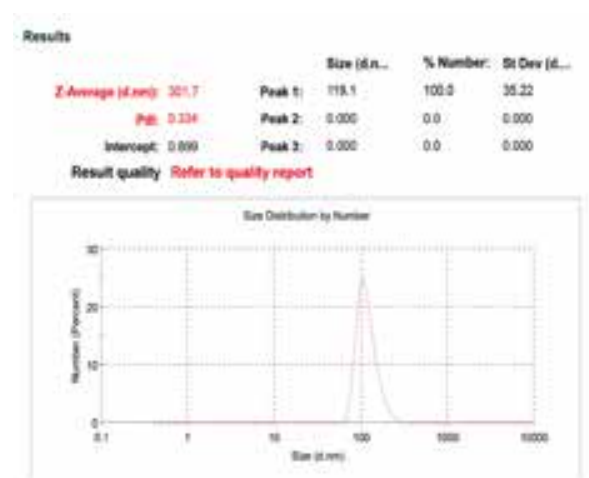

a

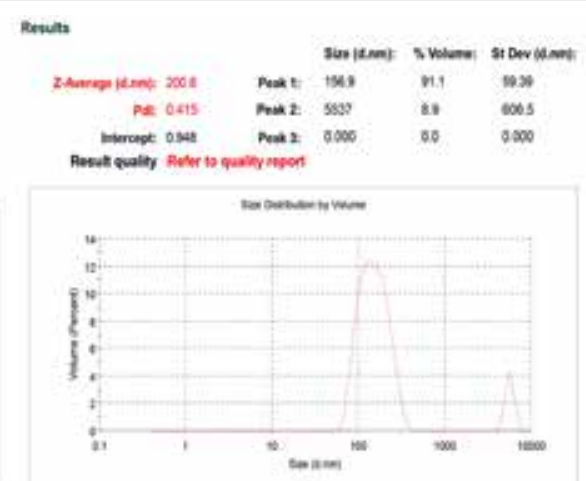

b

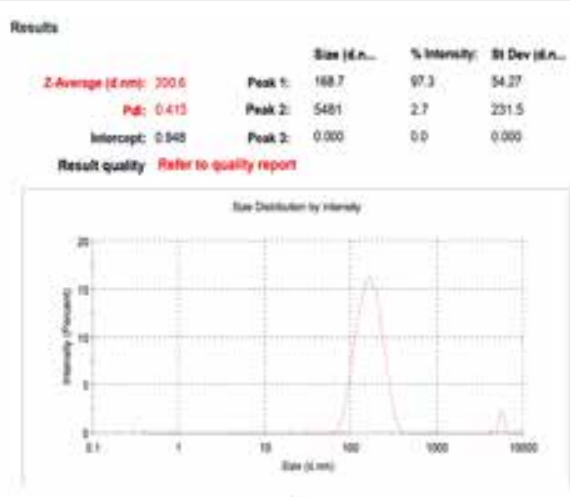

c

Figure 2. Particle size distribution of the optimized formulation; $50 \mathrm{mg} C L X$ in $50 \mathrm{mgPLGA} / 20 \mathrm{~mL}$

CLX: Celecoxib, PLGA: Poly (lactic-co-glycolic acid) 


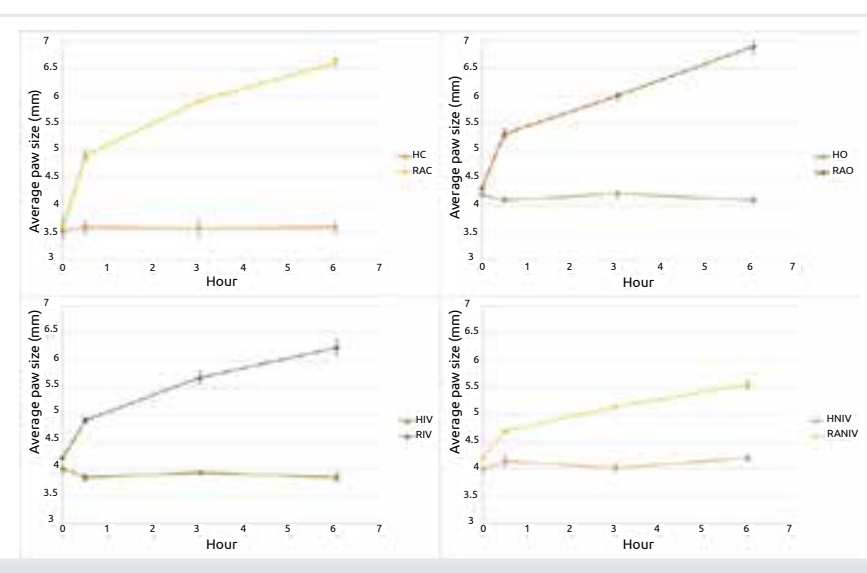

Figure 3. The size of the infected hint paw during the observations for 6 hours. The graphics are classified according the drug application groups: Up-left; Control, up-right: Orally administered Celebrex, bottom-left: intravenous CLX and bottom-right: intravenous nano-CLXPLGA (H: Healthy and RA: Rheumatoid Arthritis)

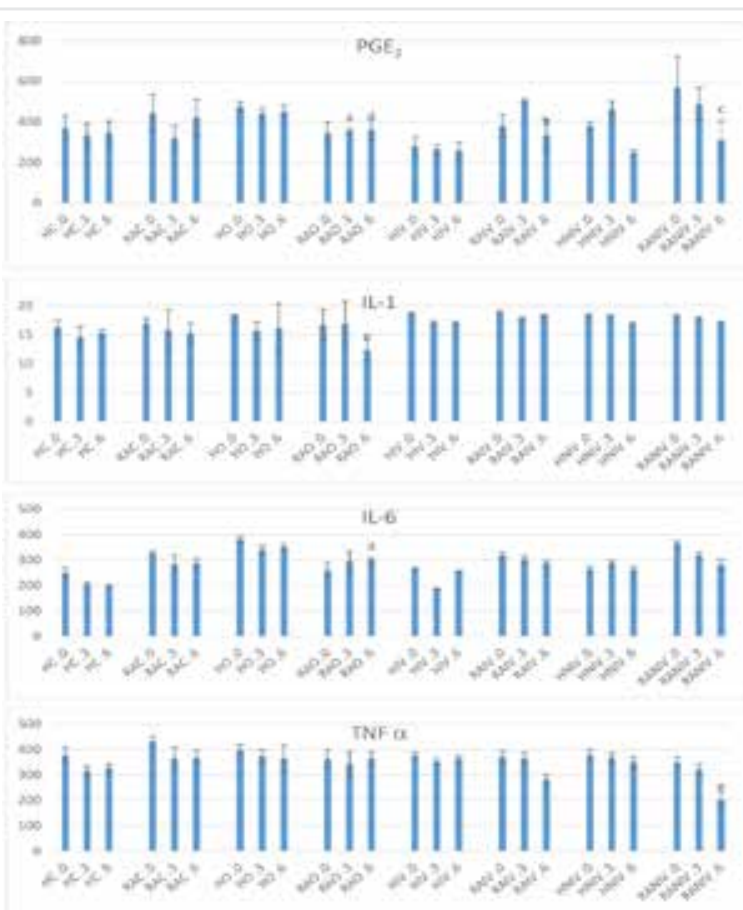

Figure 4. The variations in the blood ratios of PGE2, IL 1, IL6 and TNF- $\alpha$. HC: healthy control Group, RAC: rheumatoit artritis control group, HO: healthy rats treated with oral celebrex, RAO: rheumatoit artritis model, treated with oral celebrex, HIV: healthy rats treated with intravenous celebrex, RAIV: rheumatoit artritis model treated with intravenous celebrex, HNIV: healthy rats treated with intravenous Nano-CLX-PLGA, RAIV: rheumatoit artritis model treated with Nano-CLX-PLGA. A: significantly lower than RANIV, b: significantly lower than RAO.3 and lower tan RIV.6 ( $p<0.10)$, c: Significant decrease compared to HNIV.6, $\mathrm{d}$ : significant decrease compared to HO. 6, e: significant decrease compared to HO.6, d: significant decrease compared to HO.6, e: significant decrease compared to HNIV.6 and RAIV 6 . (in each group $p<0.05$ or $p<0.10$ ) between the group which orally received CLX and the control group in terms of the level of biological indicators. According to other results, differences between $\mathrm{HC}$ and RAC groups were similar to differences between $\mathrm{HO}$ and $\mathrm{RAO}$ groups. Except that, RAO group showed significant decrease compared to $\mathrm{HO}$ ( $p>0.05$ ), in terms of IL-6 and PGE2.

\section{Results of Release Study of CLX-PLGA}

The release profile of CLX from nano-PLGA micelles is shown in Figure 5. Almost 70\% of the drug was released within 6 hours with an accelerated behavior which was expected from an antiinflammatory agent. After 6 hours, the release profile showed a very fast increase, probably due to disruption in the structure of the nano-micelle in the dialysis membrane.

\section{Drug Loading Capacity}

As it could be seen in particle sizing results, the optimized formulation was determined as the $50 \mathrm{mg}$ CLX uploaded to 50 g PLGA. Thus, this formulation was injected to HPLC to detect the amount of drug inside polymeric micelles and at the aqueous media. EE\% was calculated as:

\section{Stability Studies}

Figure 6 shows the results of stability studies made on CLXPLGA at two different incubation conditions. While incubation at room temperature caused a short period of stability, incubation at $-20{ }^{\circ} \mathrm{C}$ resulted in obtaining perfect stability, revealed with keeping almost all drug inside of the NDDS.

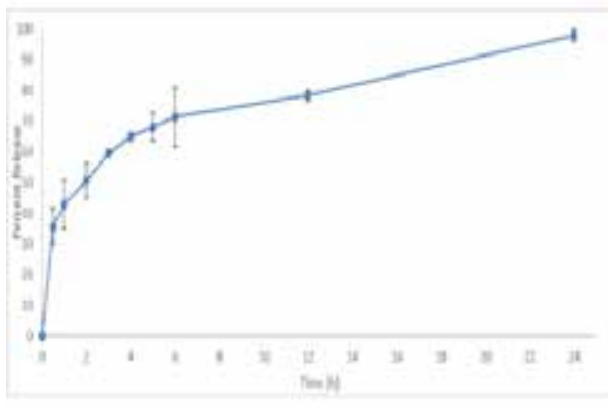

Figure 5. Celecoxib Loaded Nano-PLGA release Results

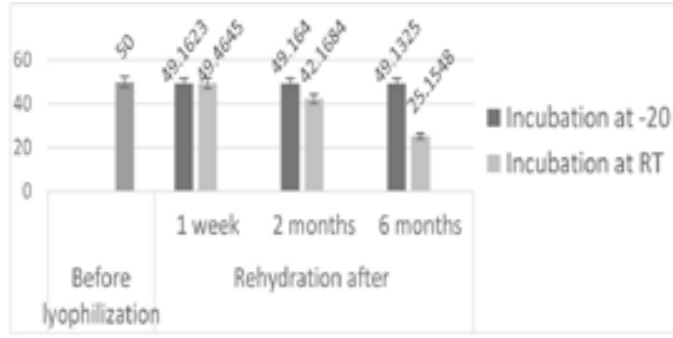

Figure 6. Stability study results of optimized CLX-PLGA formulation containing $50 \mathrm{mg} \mathrm{CLX}$, rehydrated after lyophilization and incubation at RT or $-20^{\circ} \mathrm{C}$ (RT: room temperature) 


\section{Discussion}

Nonsteroidal antiinflammatory drugs (NSAIDs) were announced in the 1960s and became the most widespread prescribed class of drugs in the world, with over 100 million prescriptions issued per year in the United States alone (20). They were used especially for the treatment of the inflammation, mostly arthritis, and pain. NSAIDs inhibit the COX which decreases the inflammation and the pain through the prostaglandin inhibition. But, the COX enzyme is also existing in gastric mucosa where it stimulates the gastro-protective prostaglandins. The identification of two isoforms, COX-1 and COX-2, and recognition of direct relationship between COX-1 inhibition and gastrointestinal system side effects, resulted in development of selective COX-2 inhibitors that offered potential to maintain efficacy while decreasing the GI adverse effects (21). Consequently, administration of the NSAIDs unavoidably leads to a lack of prostaglandins which are essential for the physiological functions. Therapeutic and side effects of this class of anti-inflammatory drugs are strongly related to their biochemical mechanism of action (8). As a result, long term NSAID users suffer from a high incidence of GI irritation. The risk of death or hospitalization from a GI adverse event varies from $1.3 \%$ to $1.6 \%$ per year in patients with RA (22). These rare, however potentially serious GI side effects of NSAIDs have become a major healthcare problem (23).

There are several nanotechnological administrations. Among them, the treatment, diagnosis, monitoring and control of the biological systems have lately been referred as "nano-medicine" by the National Institute of Health (Bethesda, MD, USA). We can easily say that the "targeting" property of nanotechnologymanaged particles is the most attractive subject in this area.

The size of the $50 \mathrm{mg}$ CLX incorporated with $50 \mathrm{mg}$ PLGA/20 $\mathrm{mL}$ nano-formulation is under $200 \mathrm{~nm}$ and is very suitable for targeting site of inflammation and also for studying the toxic dose of CLX due to the high drug loading efficacy. The release properties of this nano-formulation is long enough to carry the drug molecule in NDDS and is accelerated enough to show the fast effect in urgent cases however according to the in vivo results, the commercially available oral formulation of Celebrex shows much faster results.

Previously, Harirforoosh et al. (24) have prepared nano-PLGACLX to assess its pharmacodynamics and pharmacokinetics in rats. However, no efficacy studies were done in this research. Their results were in great accordance with our results. Since the surfactant used in their formulation was different, the particle sizes were not comparable however, the increasing ratio of CLX in serum showed similarity to our release results. Interestingly, in the study of Harirforoosh et al. (24) there was no inflammation site and the NDDS releasedthe drug within the blood flow similar to that of conventional drug and in this condition no difference between PGE2 ratios were observed. This could be an evidence of the success obtained by our group in targeting the inflammation site and inhibiting PGE2 more significantly by nano-CLX-PLGA. Similarly, Amrite et. al (14) showed 40\% reduction in PGE2 by local administration of CLX-PLGA microparticles (diameter= $1140 \pm 15 \mathrm{~nm}$ ), containing $14.93 \% \pm 0.21 \%$ of CLX. In another study, CLX-PLGA was prepared to study its antitumor activity against brain tumor cells. In this study, the drug/ polymer ratio was completely in accordance with the results obtained by our group, where acetone was used as solvent, however the particle size was different due to the applied preparation method. Since this study was an in vitro study, the targeting property of CLXPLGA was not revealed however, the release study which was similar to ours, showed very similar profile (25).

The biochemical experiments of the nano-CLX effect prove that CLX has elongated and sustained efficacy on inflammation while incorporated with micelles comparing to the conventional CLX; CLX. Comparing the paw size of the healthy and inflammated animal groups showed that CLX which was given by gavage and also administered intravenously showed almost the same efficacy. It was showed that PGE2 which is directly related to COX-2 enzyme inhibition was decreased longer with intravenous nanoCLX formulation than Celebrex.

In an overview, our results are cohesive and correlated with the hypothesis that the counted parameters are being decreased in blood and therefore increased in the tissues.

In general, differences occurred in the blood levels of IL-1 and IL-6 were not significant, probably due to their "pro" inflammation role. However, comparing IL-1 levels in the RAO and RANIV revealed that RAO group had a dramatic decrease within 3 hours. The results obtained from the IL- 6 and IL-1 were correlated to each other.

\section{Conclusion}

As a conclusion, according to the results obtained from this study, it is proved that the suggested nano-CLX-PLGA formulation is able to target the inflammated tissues in the systemic blood flow and have a better selective COX-2 inhibition activity. It is obvious that the results obtained from RAO, RAIV and RANIV groups are obtained from a single dosage administration and to achieve complete response, multiple doses are necessary.

\section{Ethics}

Ethics Committee Approval: The Bezmialem Vakıf Universty Experimental Animals Ethics Committee received confirmation from the experimental animals used. Numbered 2016-241.

Informed Consent: N/A.

Peer-review: Externally peer-reviewed.

\section{Authorship Contributions}

Concept: F.B., Design: F.B., A.K., Data Collection or Processing: Ş.E.K.T., Z.E., A.Ş.B., A.S.K., Analysis or Interpretation: F.B., A.K., H.A., H.N.K., Literature Search: F.B., Z.E., A.Ş.B., A.S.K., Writing: F.B.

Conflict of Interest: No conflict of interest was declared by the authors. 
Financial Disclosure: The authors declared that this study received no financial support.

\section{References}

1. Peakman M,Vergani D. Basic and Clinical Immunology. Elsevier Health Sciences;2009.

2. Simon LS, Lanza FL, Lipsky PE, Hubbard RC, Talwalker S, Schwartz BD et al. Preliminary study of the safety and efficacy of SC-58635, a novel cyclooxygenase 2 inhibitor: efficacy and safety in two placebo-controlled trials in osteoarthritis and rheumatoid arthritis, and studies of gastrointestinal and platelet effects. Arthritis Rheum 1998;41:1591-602.

3. Katori M, Majima M. Cyclooxygenase-2: its rich diversity of roles and possible application of its selective inhibitors. Inflamm Res 2000;49:367-92

4. de Gaetano G, Donati MB,Cerletti C. Prevention of thrombosis and vascular inflammation: benefits and limitations of selective or combined COX-1, COX-2 and 5-LOX inhibitors. Trends Pharmacol Sci 2003;24:245-52.

5. Qiao W, Wang B, Wang Y, Yang L, Zhang Y, Shao P. Cancer therapy based on nanomaterials and nanocarrier systems. J Nanomater 2010;2010:7.

6. Maeda H. Macromolecular therapeutics in cancer treatment: the EPR effect and beyond. J Control Release 2012;164:138-44.

7. Nair LS, Laurencin CT. Biodegradable polymers as biomaterials. Prog Polym Sci 2007;32:762-98.

8. Tian H, Tang Z, Zhuang X, Chen X, Jing X. Biodegradable synthetic polymers: preparation, functionalization and biomedical application. Prog Polym Sci 2012;37:237-80.

9. Gupta AP, Kumar V. New emerging trends in synthetic biodegradable polymers-Polylactide: A critique. Eur Polym J 2007;43:4053-74.

10. Mohamed F, van der Walle CF. Engineering biodegradable polyester particles with specific drug targeting and drug release properties. J Pharm Sci 2008;97:71-87.

11. Muthu MS. Nanoparticles based on PLGA and its co-polymer: An overview. AJP 2019;3:266-73.

12. Bahadori F, Kocyigit A, Onyuksel H, Dag A, Topcu G. Cytotoxic, Apoptotic and Genotoxic Effects of Lipid-Based and Polymeric Nano Micelles, an In Vitro Evaluation. Toxics 2017;6:7.

13. Banerjee A,Onyuksel $H$. Human pancreatic polypeptide in a phospholipid-based micellar formulation. Pharm Res 2012;29:1698711.
14. Amrite AC, Ayalasomayajula SP, Cheruvu NP, Kompella UB. Single periocular injection of celecoxib-PLGA microparticles inhibits diabetes-induced elevations in retinal PGE2, VEGF, and vascular leakage. Invest Ophthalmol Vis Sci 2006;47:1149-60.

15. Bahadori F, Topçu G, Eroğlu MS, Önyüksel H. A new lipidbased nano formulation of vinorelbine. AAPS PharmSciTech 2014;15:1138-48.

16. Emami J, Fallah R, Ajami A. A rapid and sensitive HPLC method for the analysis of celecoxib in human plasma: application to pharmacokinetic studies. DARU 2008;16:211-17.

17. Baboota S, Faiyaz S, Ahuja A, Ali J, Shafiq S, Ahmad S. Development and validation of a stability-indicating HPLC method for analysis of celecoxib (CXB) in bulk drug and microemulsion formulations. Acta Chromatogr 2007; 18:116.

18. Wong HL, Bendayan R, Rauth AM, Wu XY. Development of solid lipid nanoparticles containing ionically complexed chemotherapeutic drugs and chemosensitizers. J Pharm Sci 2004;93:1993-2008.

19. Cakal B, Akoz AG, Ustundag Y, Yalinkilic M, Ulker A, Ankarali H. Red cell distribution width for assessment of activity of inflammatory bowel disease. Dig Dis Sci 2009;54:842-47.

20. Bello AE, Holt RJ. Cardiovascular risk with non-steroidal antiinflammatory drugs: clinical implications. Drug Saf 2014;37:897902.

21. Whittle B. COX-1 and COX-2 products in the gut: therapeutic impact of COX-2 inhibitors. Gut 2000;47:320-25.

22. Fries JF. NSAID gastropathy: the second most deadly rheumatic disease? Epidemiology and risk appraisal. J Rheumatology Suppl 1991;28:6-10.

23. Silverstein FE, Graham DY, Senior JR, Davies HW, Struthers BJ, Bittman RM, et al. Misoprostol reduces serious gastrointestinal complications in patients with rheumatoid arthritis receiving nonsteroidal anti-inflammatory drugs. A randomized, double-blind, placebo-controlled trial. Ann Intern Med 1995;123:241-49.

24. Harirforoosh S, West K, Murrell D, Denham J, Panus P, Hanley G. Assessment of celecoxib poly (lactic-co-glycolic) acid nanoformulation on drug pharmacodynamics and pharmacokinetics in rats. Eur Rev Med Pharmacol Sci 2016;20:4818-29.

25. Kim T-H, Jeong Y-I, Jin S-G, Pei J, Jung T-Y, Moon K-S, et al. Preparation of polylactide-co-glycolide nanoparticles incorporating celecoxib and their antitumor activity against brain tumor cells. Int J Nanomedicine 2011;6:2621-31. 